\title{
LEAUCH: low-energy adaptive uneven clustering hierarchy for cognitive radio sensor network
}

\author{
Errong Pei ${ }^{{ }^{*}}$, Haozhe Han ${ }^{1}$, Zehua Sun ${ }^{1}$, Bin Shen ${ }^{1}$ and Tianqi Zhang ${ }^{2}$
}

\begin{abstract}
The integration of wireless sensor network (WSN) and cognitive radio (CR) technology enables a new paradigm of communication: cognitive radio sensor networks (CRSN). The existing WSN clustering algorithm cannot consider the advantage of channel resource brought by CR function in CRSN, and the CR network (CRN) clustering algorithm is designed based on the infinite energy nodes; thus both algorithms cannot operate with energy efficiency in CRSN. The paper proposes a low-energy adaptive uneven clustering hierarchy for CRSN, which can not only consider the advantage of the channel resource in reducing the energy consumption but also employ uneven clustering method for balancing the energy consumption among the cluster heads under multiple hops transmission means. Simulation results show that compared with the existing several typical clustering algorithms including WSN and CRSN clustering algorithms, low-energy adaptive clustering hierarchy (LEACH), HEED, energy-efficient unequal clustering (EEUC), cognitive LEACH (CogLEACH), and distributed spectrum-aware clustering (DSAC), the proposed algorithm can not only efficiently balance the energy consumption among cluster heads and network load in CRSN but also remarkably prolong the network lifetime.
\end{abstract}

Keywords: Cognitive radio sensor network; Channel resource; Uneven clustering; Energy consumption

\section{Introduction}

The existing wireless sensor network (WSN) operates in the public unlicensed spectrum band, which has become increasingly crowd because of the emergence of the vast wireless communication technologies. Meanwhile, the increasing serious mutual interference caused by the coexistence of heterogeneous wireless systems in the public unlicensed spectrum band has become a bottleneck problem which greatly limits the further development of WSN.

In order to solve the above both problems in WSN, the cognitive radio sensor networks (CRSN) is proposed, where implement dynamic spectrum access can be used [1-3]. Introducing cognitive radio (CR) technology into WSN makes the sensor nodes possess the ability of spectrum sensing. WSN can thus operate over the idle licensed spectrum band, which can not only reduce the collision

*Correspondence: cqpeier@qq.com

1 Engineering Research Center of Communication Network Testing

Technologies, Chongqing University of Posts and Telecommunications, No. 2,

Chongwen Rd, Nan'an Dist, Chongqing 400065, China

Full list of author information is available at the end of the article probability of channels but also increase spectrum utilization and enlarge the operate spectrum band. The network throughput can further be increased, and the communication delay can also be shortened [4].

Since a large number of sensor nodes with limited energy may be randomly deployed in harsh environments and operate by forming a network in an ad hoc manner. Such a scenario requires an energy-efficient routing protocol that accounts for scalability. Clustering is shown to achieve such constraints and generally enlarges the network lifetime [5].

However, CRSN brings a new technical challenge in terms of its routing algorithm. On the one hand, the existing routing strategies in CR network (CRN) mainly focus on overcoming the spectrum scarcity and increasing the spectrum utilization by providing the combination scheme of the spectrum and route. However, they fail to consider the energy scarcity problem and hardware limitation problem inherited from the traditional WSN [6-9]. On the other hand, the existing clustering routing algorithms in non-CR WSN mainly focus on minimizing

\section{Springer}

(c) 2015 Pei et al: licensee Springer. This is an Open Access article distributed under the terms of the Creative Commons

Attribution License (http://creativecommons.org/licenses/by/4.0), which permits unrestricted use, distribution, and reproduction in any medium, provided the original work is properly credited. 
energy consumption in nodes. However, they do not consider the spectrum sensing and spectrum management problem [10-13]; thus, they cannot exploit the advantage of spectrum resource brought by CR function in reducing energy consumption, and further, they cannot efficiently operate in CRSN.

Recently, CRSN has attracted much attention of researches from the world, and some literatures including routing algorithms are also published [6,14-24]. Taking account of the shortcomings of the existing clustering routing algorithm in WSN and CRN, the paper proposes a low-energy adaptive uneven clustering hierarchy (LEAUCH) for CRSN, which can not only consider the advantage of channel resources brought by cognitive function in CRSN but also exploit the uneven clustering method based on the channel resources. More specially, in the proposed algorithm, the number of idle channels of each node is taken as its weight and the nodes with more idle channels are elected as candidate cluster head $(\mathrm{CH})$ nodes. Based on the idea of the uneven clustering method, there are fewer members in the clusters near the sink. In this way, the energy of $\mathrm{CH}$ near sink can be saved, and further more energy can be used for forwarding data, which can balance energy consumption among CHs under multiple hops transmission means in CRSN. In addition, when selecting the next hop node, the $\mathrm{CHs}$ consider not only its relative distance to the sink but also residual energy in the candidate nodes. Experimental results show that the proposed algorithm can not only be suitable for the CRSN, effectively balance the energy consumption in $\mathrm{CHs}$ under multiple hop transmission means, but also optimize the energy consumption of each node in the network, and further remarkably prolong the network lifetime.

The paper is organized as follows. Section 2 presents related work. Section 3 presents network model and LEAUCH algorithm. Section 4 is a performance analysis of LEAUCH. Section 5 is a simulation analysis of the proposed algorithm. The last section is a conclusion of the paper.

\section{Related work}

Low-energy adaptive clustering hierarchy (LEACH) [25] is regarded as the most representative traditional algorithms. However, it can be only suitable for WSN operating in the unlicensed spectrum band, and thus, it cannot operate with energy efficiency in CRSN.

The literature [14] proposed a distributed spectrumaware clustering (DSAC) algorithm based on the traditional K-means clustering algorithm with group-wise constraint. The algorithm initially takes each node as $\mathrm{CHs}$ and then merges $\mathrm{CHs}$ in each iteration until the number of $\mathrm{CHs}$ reaches a theoretically optimal number. However, a large number of information is intensively exchanged between nodes and $\mathrm{CHs}$, which lead to the great waste of the energy in the nodes.

The literature [15] proposed a cognitive LEACH (CogLEACH) for CRSN that uses the number of vacant channels as a weight in the probability of each node to become a $\mathrm{CH}$ and that can prolong the network lifetime compared with LEACH algorithm. However, the algorithm does not consider the balance of energy consumption among $\mathrm{CHs}$ under multiple hops transmission means, which may lead to the premature death of the nodes near the sink because of their excessive energy consumption ( $\mathrm{CHs}$ near the sink need to frequently forward data to the sink).

The literature [22] proposed a spectrum-aware clusterbased energy-efficient multimedia (SCEEM) routing protocol for CRSNs, which can support the quality of service (QoS) and energy-efficient routing by limiting the participating nodes in route establishment. The proposed protocol in the literature is thus a cross layer routing protocol and only suitable for wireless multimedia sensor networks (WMSNs) application scenario, which comprised of sensor devices equipped with audio and visual information collection modules, can have the ability to retrieve multimedia data, store or process data in real-time, correlate and fuse multimedia data originated from heterogeneous sources, and wirelessly transmit collected data to desired destinations. Moreover, WMSNs are designed for those real-time applications which demand strict deadline, low delay, high throughput, and reliability as well as those non-real time applications which require high or medium bandwidth, loss intolerance, etc. However, most deployed WSNs measure physical phenomena like temperature, pressure, humidity, or location of objects. In general, most of those applications have low bandwidth demands and are usually delay tolerant. Therefore, the proposed protocol in the literature cannot be suitable for the CRSNs.

The literature [16] proposed an event-driven clustering algorithm. The qualified nodes are determined based on the distance from sensor nodes to the event occurrence point and the sink. CHs are selected among the qualified nodes according to node degree, available channels, and the distance to the sink in their neighborhood. The clusters in the scenario are immediately dismissed after finishing data transmission, and all nodes enter the sleeping state again in order to save the energy. Therefore, the proposed algorithm in the literature is only confined to event-driven CRSN, which cannot be suitable for other scenarios such as the time-triggered CRSN scenario.

In order to prolong the network lifetime, prior research works mainly focus on balancing energy consumption among nodes as cluster members (CMs). However, they neglect the problem of balancing energy consumption among $\mathrm{CHs}$ under multiple hops transmission means. 
In energy-efficient unequal clustering (EEUC) algorithm [17], the authors proposed an uneven-sized clustering method to balance the energy consumption among $\mathrm{CHs}$. However, the EEUC algorithm does not take into account the advantage brought by CR technology in CRSN, so its $\mathrm{CHs}$ selection method can only apply to WSN. Apparently, the algorithm cannot efficiently operate in CRSN.

\section{Network model and algorithm description}

\subsection{Network model}

In the paper, we consider the scenario where there are $N$ randomly deployed CRSN nodes collecting data periodically and $P$ primary users (PU). Let $s_{i}$ denote the $I$ th node, and the corresponding set of nodes is denoted by $S=\left\{s_{1}, s_{2}, \cdots, s_{N}\right\}$. Let $C=\{1,2, \cdots, m\}$ denote the available channels in the network and $C_{i}$ the idle channels available to node $s_{i}$. We assume:

1. The sink is located outside the square observation area. Once deployed, CRSN node and the sink will no longer be moved;

2. All nodes are homogeneous and possess the function of information fusion. Each node has a unique identification (ID);

3. Each node has the ability of spectrum sensing and can correctly detect the available channels in the surroundings;

4. All nodes can adjust its transmit power to save energy based on its distance from the receiver;

5. The entire network has a network-wide common control channel (CCC) [26].

The CR technology is introduced into sensor nodes in CRSN, so the traditional LEACH algorithm must be improved to adapt the new scenario. For purpose of making the number of CMs near the sink relatively small, the CRSN is divided into many uneven clusters based on the determined $\mathrm{CHs}$, as shown in Figure 1. In Figure 1, the

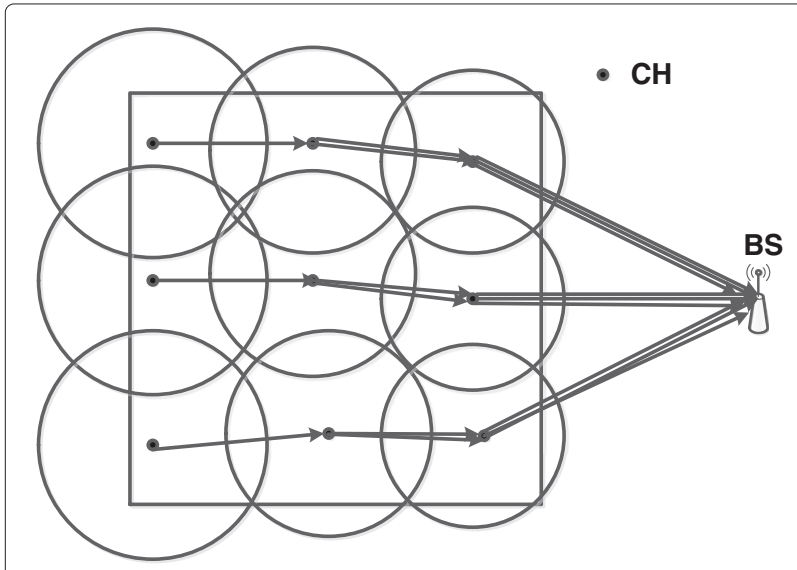

Figure 1 Uneven clustering routing algorithm. circles of different sizes represent the different competitive ranges of the $\mathrm{CHs}$ and the lines with arrow represent multiple hop data transmission among $\mathrm{CHs}$.

\subsection{Determination of the candidate $\mathrm{CHs}$}

In the regular LEACH, $P_{i(t)}$ is chosen such that $E\{\# C H s\}=k$, where [14]

$$
P_{i(t)}= \begin{cases}\frac{k}{N-k *\left(r \bmod \frac{N}{K}\right)} & : C_{i}(t)=1 \\ 0 & : C_{i}(t)=0\end{cases}
$$

where $N$ denotes the total number of nodes, $k$ denotes the desired number of $\mathrm{CHs}$ (on average) per round, $r$ denotes the current round number, and $C_{i}(t)$ is an indicator function determining whether or not node $i$ has been a $\mathrm{CH}$ for the most recent $r \bmod (N / K)$ rounds. This formula of $P_{i(t)}$ maintains that each node is selected as a $\mathrm{CH}$ once per cycle of operation and thus balancing the load between nodes and extending time of the first node death.

The number of idle channels detected in each node is considered as an important factor in choosing $\mathrm{CHs}$ in the paper. If node $i$ detects more idle channels than node $j$, it has more opportunity to find a common channel with nearby nodes, which may result in more opportunity to set up a cluster with a common channel.

A form of $P_{i(t)}$ should be constructed such that:

- $E\{\# C H s\}=k$.

- A node with more idle channels is more likely to become a $\mathrm{CH}$.

According to the literature [10], the probability of a node to become $\mathrm{CH}$ is:

$$
P_{i(t)}=\min \left(k \frac{c_{i}}{\sum_{j=1}^{N} c_{j}}, 1\right)
$$

where $c_{i}$ is the number of available channels. In the paper, the nodes with probability of $P_{i(t)}>0.4$ is selected as candidate $\mathrm{CHs}$.

\subsection{Determination of $\mathrm{CHs}$}

The nodes with $P_{i(t)}>0.4$ are selected as the candidate $\mathrm{CHs}$ and start to compete for $\mathrm{CHs}$; other nodes enter sleeping mode until the end of the competition. Let $s_{i}$ be one candidate $\mathrm{CH}$, and its competition radius $R_{c}$ can be calculated on the basis of the distance from the sink. During the competition, if $s_{i}$ wins, then all other candidate nodes within its competition radius $R_{c}$ quit the competition for $\mathrm{CHs}$.

Figure 2 is the topology structure of candidate $\mathrm{CHs}$. In Figure 2, the circles with different sizes represent competition radius of candidate $\mathrm{CHs}$. In accordance with the rule of determining $\mathrm{CHs}, s_{1}$ and $s_{2}$ can become final $\mathrm{CHs}$ at the same time; however, $s_{3}$ and $s_{4}$ cannot become final 


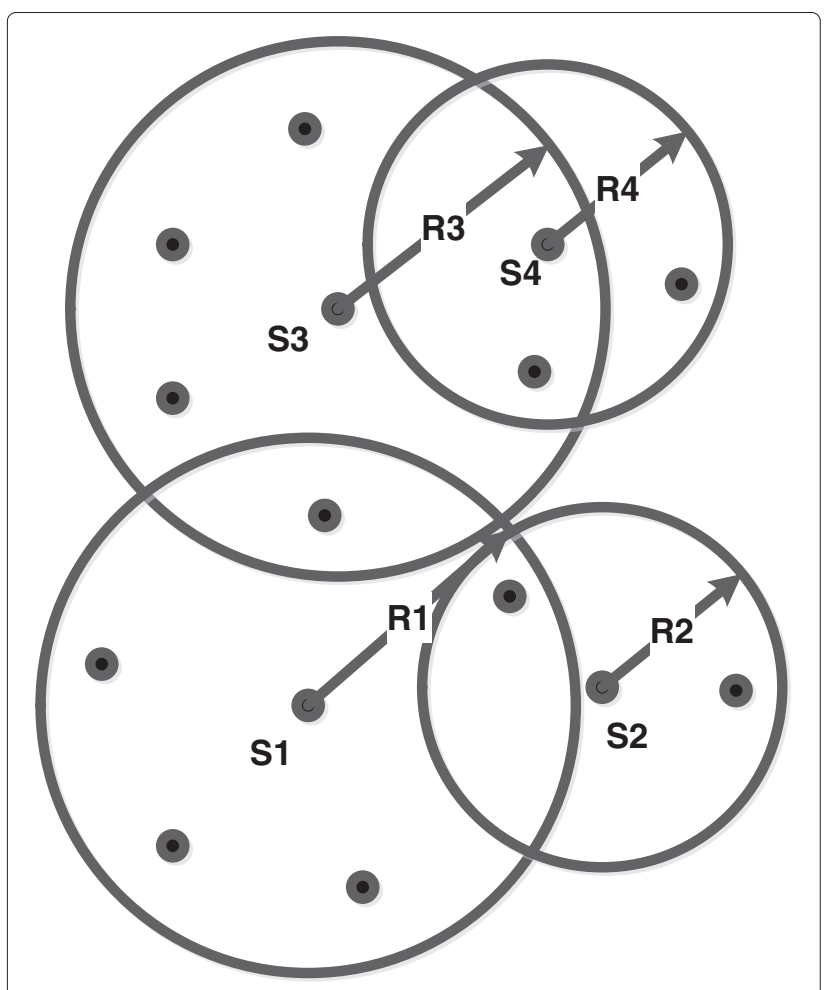

Figure 2 Competition radiuses of the candidate $\mathrm{CHs}$.

$\mathrm{CHs}$ at the same time because $s_{4}$ falls in the range of the competition radius of $s_{3}$.

In the proposed algorithm, the clusters near the sink have short competition radius and fewer CMs, so that their $\mathrm{CHs}$ can use more energy to communicate among clusters and further balance the energy consumption among $\mathrm{CHs}$.

Let $R_{c}^{0}$ be the maximum value of competition radius of candidate $\mathrm{CHs}$, then the competition radius of the candidate $\mathrm{CHs}$ can be determined by $(1-c) R_{c}^{0}$, where $c$ is the parameter used for controlling its value and $c \in[0,1]$. Therefore, the competition radius $R_{c}$ of a candidate node $S_{i}$ can be determined by [17]:

$$
R_{c}=\left(1-c \frac{d_{\max }-d\left(s_{i}, B S\right)}{d_{\max }-d_{\min }}\right) R_{c}^{0}
$$

where $d_{\max }$ and $d_{\text {min }}$ denote the maximum and minimum of the distance from the nodes to the sink respectively, and $d\left(s_{i}, B S\right)$ denotes the distance between the node $s_{i}$ and sink. The competition radius decreases linearly with the distance from the nodes to the sink. For example, $c=1 / 4$, the range of the competition radius is $3 / 4 R_{c}^{0} \sim R_{c}^{0}$.

Definition 1. In $\mathrm{CHs}$ competition algorithm of LEAUCH, the set of adjacent $\mathrm{CH}$ s of the candidate $\mathrm{CH} s_{i}$ is denoted by $S_{s_{i}}$ and

$$
S_{s_{i}}=\left\{s_{j} \mid s_{j} \text { is candidate } \mathrm{CH}, d\left(s_{i}, s_{j}\right)<\max \left(R_{s_{i}}, R_{s_{j}}\right)\right\}
$$

In the algorithm, each candidate node broadcasts their own competition message in the same power including node ID, competition radius $R_{c}$, and current residual energy, and the broadcast radius can be set to $R_{c}^{0}$ in order to save energy.

After receiving the broadcast message, each candidate $\mathrm{CH}$ forms its adjacent candidate $\mathrm{CH}$ set and then decides whether it becomes the $\mathrm{CH}$ or not. $\mathrm{CH}$ s can be decided according to the following step:

1. The node $s_{i}$ with the highest residual energy in $S_{S_{i}}$ become $\mathrm{CH}$, then broadcast it;

2. If node $s_{i}$ receives the winning message from $s_{j}$ $\left(s_{j} \in S_{s_{i}}\right), s_{i}$ quits the competition and broadcasts the quit message;

3. If node $s_{i}$ receives the quit message from $s_{j}\left(s_{j} \in S_{s_{i}}\right)$, then $s_{i}$ will remove $s_{j}$ from the set of its adjacent $\mathrm{CHs}$.

\subsection{Clusters establishment}

Nodes that have not participated in the election will wake up from sleep after the $\mathrm{CH}$ s are determined and join their own clusters according to the following steps.

1. Node $s_{i}$ is one of the $\mathrm{CHs}$ in a round, and $C_{i}$ is the idle channel detected by node $s_{i}$. Then, $\mathrm{CH} s_{i}$ broadcast their own message after winning the election, including ID and available channel lists;

2. Node $C_{j}$ within the range of $s_{i}$ has one or more same element with $C_{i} . s_{j}$ sends messages including ID and available channel to request to join the cluster once it receives the $\mathrm{CH}$ information of $s_{i}$;

3. $s_{i}$ records IDs and available channels of requesting nodes and selects the $C_{i q}$, a common channel that most requesting nodes and $s_{i}$ share, then broadcast it;

4. Ordinary nodes decide to join the cluster in which the node spends the minimum energy in communicating with $\mathrm{CH}$, then sends joining messages to inform the $\mathrm{CH}$.

\subsection{Stabilization phase}

CMs in the cluster receive slot time which is distributed from $\mathrm{CHs}$ by TDMA and then transmits collected data to the $\mathrm{CHs}$ in turn. The $\mathrm{CHs}$ fuse the collected data from CMs and forward to the sink.

\section{Experimental results and analysis}

In the section, the simulation experiments on the proposed algorithm are conducted using MATLAB. The energy consumption model is derived from the literature [7]. This experiment makes a comparison between the proposed LEAUCH and the other four algorithms, LEACH [25], DSAC [14], cognitive LEACH (CogLEACH) 
[15], and EEUC [17], in terms of $\mathrm{CH}$ energy consumption, network load balance, and network lifetime.

In the paper, we consider the scenario where 200 cognitive sensor nodes are randomly distributed in an $200 \mathrm{~m} \times$ $200 \mathrm{~m}$ area, which share five available channels with five primary users. Some other simulation parameters are assumed as shown in Table 1.

$E_{\text {elec }}$ denotes the energy consumption of the circuit board when the nodes receive or transmit wireless data, $\varepsilon_{f s}$ denotes the energy amplification coefficient where $d \leq d_{0}$ in the free space, and $\varepsilon_{m p}$ denotes the energy amplification coefficient where $d>d_{0}$ in multipath attenuation model. In addition, $P_{i(t)} \geq 0.4, R_{c}^{0}=90, \mathrm{c}=0.5$.

\subsection{The comparison of total energy consumption of $\mathrm{CHs}$}

Generally speaking, $\mathrm{CHs}$ undertake more tasks than other nodes in communication, which lead to more energy consumption of $\mathrm{CHs}$ than other nodes. The performance of a clustering routing algorithm can thus be evaluated by $\mathrm{CHs}$ energy consumption, as shown in Figure 3 . Note that the 15 rounds are taken randomly from simulations.

It can be observed from Figure 3 that the $\mathrm{CH}$ energy consumption in LEAUCH is lower than that of the other protocols. $\mathrm{CHs}$ in $\mathrm{LEACH}$ are selected randomly and inefficiently, which makes $\mathrm{CHs}$ energy consumption fluctuate dramatically. In addition, the communications in and between the clusters are processed through single hop and the data do not fused necessarily, which make $\mathrm{CHs}$ energy consumption in LEACH much higher than others.

Both DSAC and CogLEACH consider the residual energy of nodes, and the distance between nodes and their information is transmitted over idle channels. It can be seen from the Figure 3 that $\mathrm{CHs}$ energy consumptions in DSAC and CogLEACH are relatively lower. However, both algorithms are based on the uniform clustering method

Table 1 Simulation parameter setting

\begin{tabular}{ll}
\hline Parameter & Value \\
\hline Parameter scene range & $200 \mathrm{~m} \times 200 \mathrm{~m}$ \\
Position of sink & $(250,100)$ \\
CRSN node number & 200 \\
Primary user & 5 \\
Available channel & 5 \\
Initial energy of & \\
Cognitive sensor node & $0.5 \mathrm{~J}$ \\
$E_{\text {elec }}$ & $50 \mathrm{~nJ} / \mathrm{bit}$ \\
$\varepsilon_{f s}$ & $10 \mathrm{pJ} / \mathrm{bit} / \mathrm{m}^{2}$ \\
$\varepsilon_{m p}$ & $0.0013 \mathrm{pJ} / \mathrm{bit}_{\mathrm{m}} \mathrm{m}^{2}$ \\
$d_{0}$ & $87 \mathrm{~m}$ \\
Data package size & $2,000 \mathrm{bits}$ \\
\hline
\end{tabular}

which leads to more energy consumption of $\mathrm{CHs}$ near the sink. At the same time, both algorithms easily produce the isolated nodes, which make them be inferior to LEAUCH.

EEUC protocol considers the distribution density of nodes and their residual energy as well as other factors when selecting $\mathrm{CHs}$, which makes the selected $\mathrm{CHs}$ be relatively ideal and distribute in a relatively even manner. The $\mathrm{CH}$ energy consumption is thus relatively low.

The LEAUCH algorithm proposed in the paper can dynamically choose the best idle channels for communications, which can decrease the possibilities of the waiting and conflict caused by competing for channels among nodes, and the $\mathrm{CHs}$ energy consumption can thus be decreased. Furthermore, The LEAUCH also employs uneven clustering method, which can also contribute to balancing the energy consumption among CHs. Therefore, LEAUCH can obtain the most performance in the CHs energy consumption.

\subsection{The comparison of network load balance}

For the purpose of improving the energy efficiency and prolonging the network lifetime, the energy consumption among $\mathrm{CHs}$ should be kept relatively balanced. Figure 4 shows the comparison of network load balance among several algorithms.

It can be seen from Figure 4 that LEAUCH and EEUC algorithms are better in load balance, LEACH protocol is the worst, and the DSAC and CogLEACH algorithms are medium.

The selection of $\mathrm{CHs}$ in $\mathrm{LEACH}$ is random, which makes its $\mathrm{CH}$ energy consumption in each round extremely unsteady. Also, $\mathrm{CHs}$ communicate with the sink directly in LEACH, which make the network load more unbalance.

In DSAC protocol, all nodes are initialized to $\mathrm{CHs}$. During the merging of $\mathrm{CHs}$, the energy consumption of information interaction between nodes and $\mathrm{CHs}$ is relatively high. Besides, node isolation problem can easily be caused. However, by introducing the cognitive function, information interaction in DSAC can operate in other available channels, which makes its network load be in medium.

The size of the clusters in CogLEACH is uniform, which may lead to the premature death of $\mathrm{CH}$ near the sink because $\mathrm{CHs}$ near the sink need to not only process data in the cluster but also forward data from other clusters. At the same time, the problem of the isolated nodes is not considered in the algorithm. Therefore, the CogLEACH obtains relatively poor performance in network load balance.

Both LEAUCH and EEUC consider the density of nodes and the distance between $\mathrm{CHs}$ and sink, which make the both algorithms have better performance in network load balance. Furthermore, considering the advantage brought 


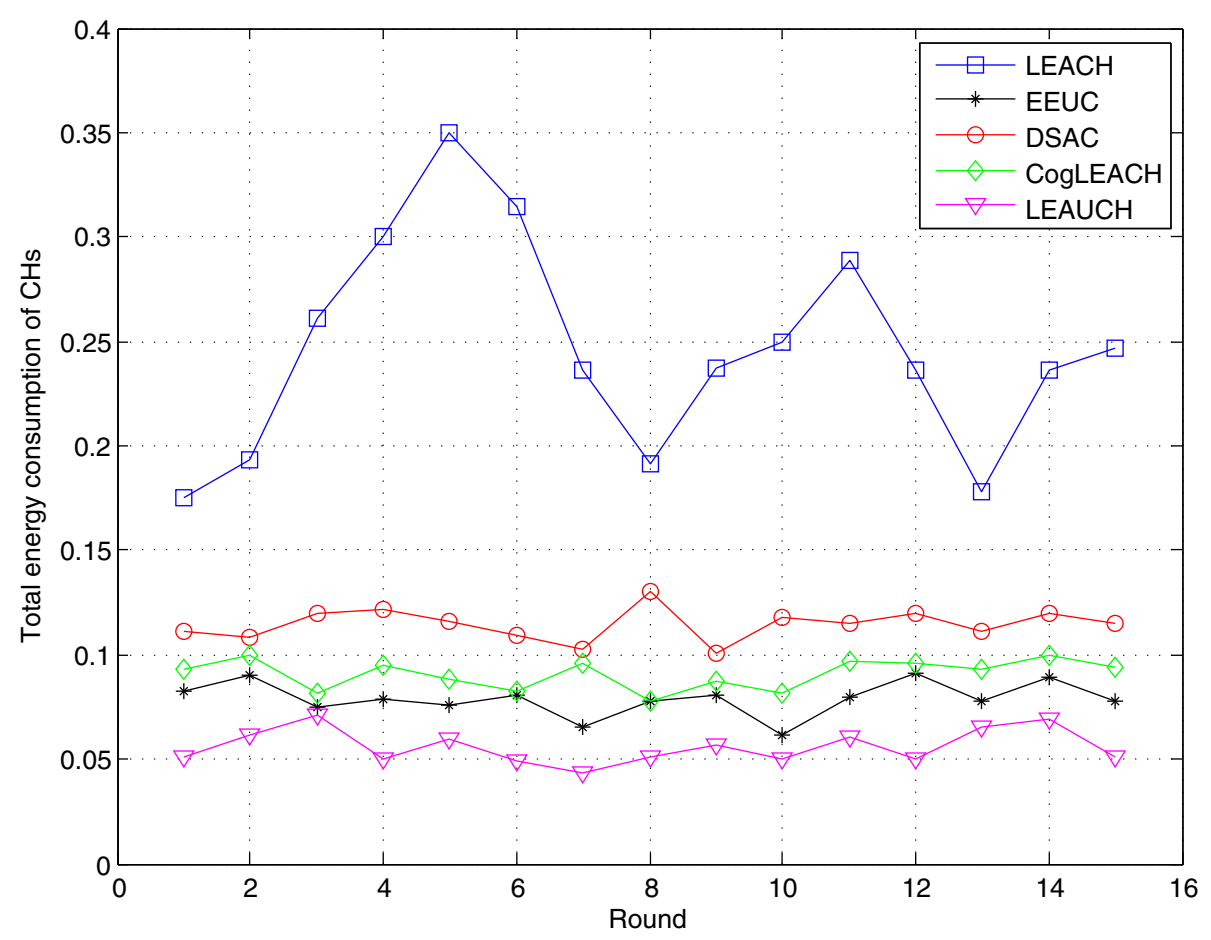

Figure 3 The comparison of total energy consumption of $\mathrm{CHs}$.

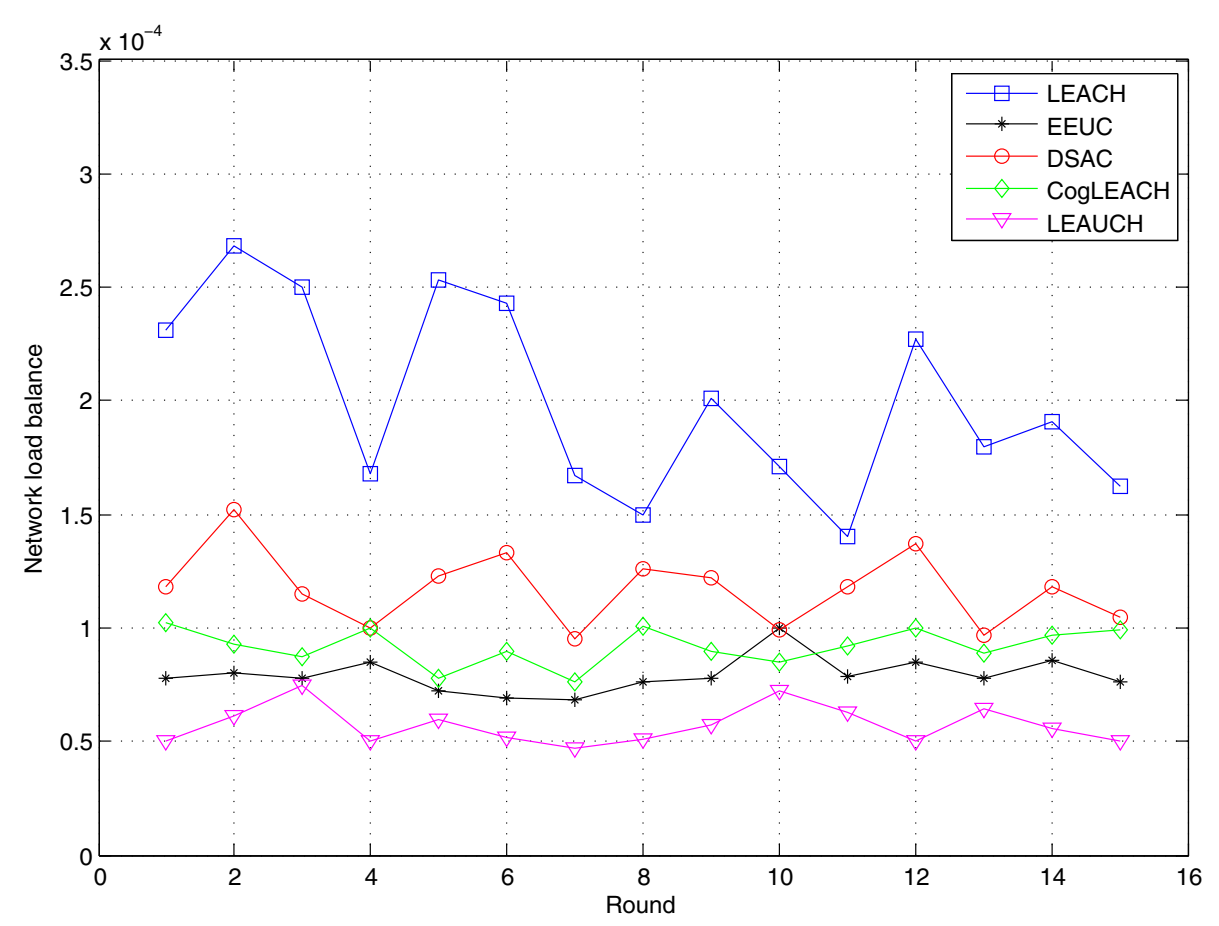

Figure 4 The comparison of network load balance. 
by CR technology in CRSN makes LEAUCH superior to EEUC.

\subsection{The comparison of network lifetime}

Figure 5 is a comparison of the number of residual living nodes among the five algorithms. It can be seen from Figure 5 that the nodes in LEAUCH remain to have longer living time than the other four algorithms. The time span between the time of the first dead node and that of the last dead node is a reflection of the energy balance among nodes. The shorter the time span is, the better the performance of energy balance among nodes is and thus the more efficient the use of the energy is. Therefore, it can be seen from Figure 5 that LEAUCH can not only prolong the network lifetime but also remain the most performance of energy consumption among all nodes. It can also be seen from the figure that the network lifetime in DSAC and $\operatorname{CogLEACH}$ is longer than other protocols due to its cognitive function.

\subsection{Performance analysis of throughput and delay}

Most deployed WSNs measure physical phenomena like temperature, pressure, humidity, or location of objects. In general, most of those applications have low bandwidth demands and are usually delay tolerant. Therefore, the proposed routing protocol in the paper mainly focuses on the improvement of energy efficiency (energy consumption of nodes and the network lifecycle), while the performance of throughput and delay can be theoretically analyzed as follows:

Throughput: Apparently, LEAUCH has a significant improvement in the obtained throughput due to the spectrum-awareness property compared with other non$\mathrm{CR}$ clustering routing protocols as $\mathrm{CogLEACH}$ in literature [9]. Nodes in non-CR WSNs may suffer from severe packet drop due to the competition for channels and interference with other systems operating on the same spectrum band, which results in a severe throughput degradation. Both of the proposed LEAUCH in the paper and $\mathrm{CogLEACH}$ are cluster-based routing protocols for CRSN; however, they have the similar data transmission process and almost same throughput.

Delay: Non-CR clustering routing algorithms operate in traditional WSN, and SNs transmit data using public spectrum band. There may exist huge amount of concurrent data transmission in public spectrum band and results in collisions, which may cause great transmission delay. The CR-based clustering routing algorithm (for example, CogLEACH and the proposed LEAUCH) in CRSN introduces cognitive function, and thus, the SNs in the CRSN can transmit data in the distributed idle channel to avoid data collision. Therefore, generally speaking, the spectrum-awareness clustering routing protocols including $\operatorname{CogLEACH}$ and the proposed LEAUCH in the paper have less delay relative to traditional clustering routing protocols, and obviously, CogLEACH and the proposed LEAUCH have almost same time delay.

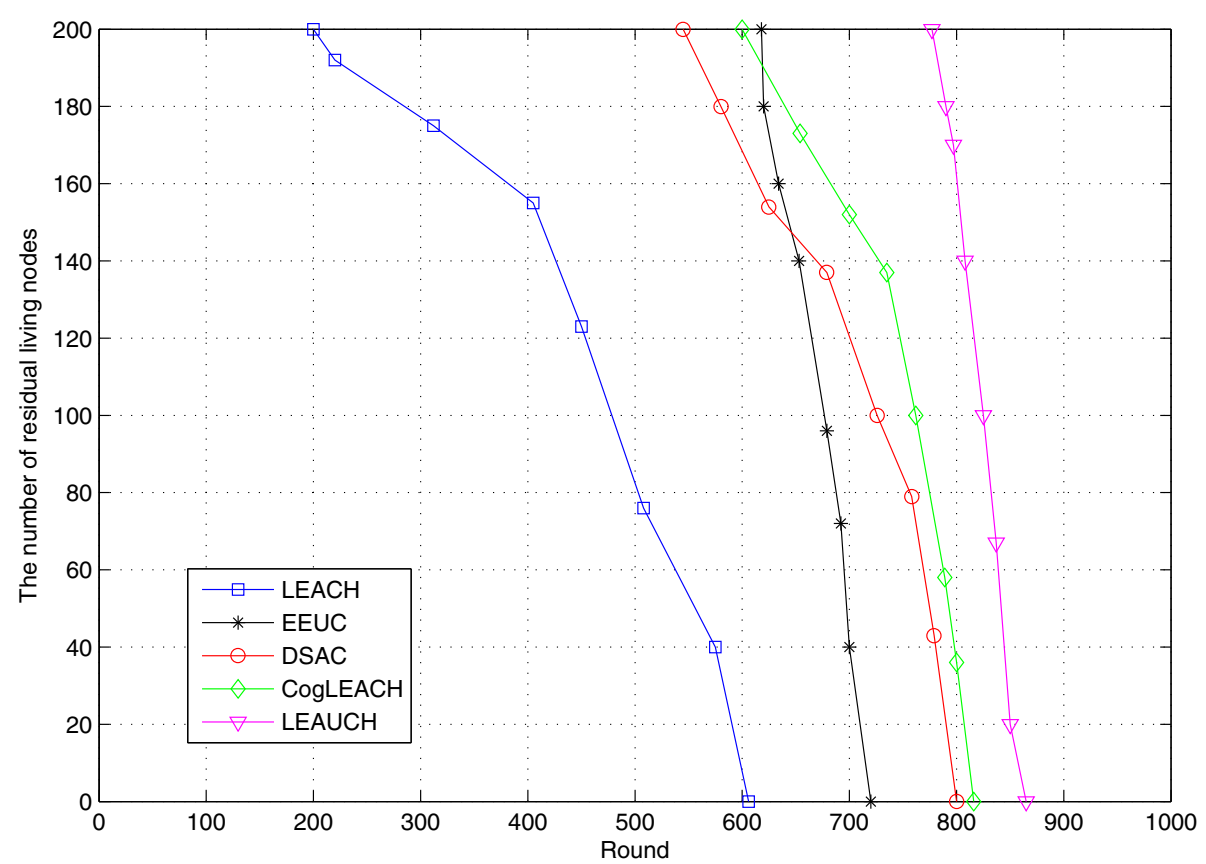

Figure 5 The comparison of the number of residual living nodes in the network. 


\section{Future works}

The proposed routing protocol in this paper mainly focuses on the improvement of energy efficiency of sensor nodes and further increase the lifetime of WSN where the sensors are triggered periodically to measure physical phenomena like temperature, pressure, humidity, or location of objects. In general, most of those applications have low bandwidth demands and are usually delay tolerant. In future works, some application scenarios which require less delay, packets loss rate, and more throughput will also be considered, and thus, more complicated network topology is further considered to evaluate their performance.

\section{Conclusions}

The paper proposed the LEAUCH algorithm CRSN, which takes the number of idle channels of each node as its weight and chooses the nodes with more idle channels as candidates $\mathrm{CHs}$. In addition, the proposed algorithm employs the uneven clustering method, which can balance energy consumption among $\mathrm{CHs}$ under multiple hops means. Experimental results show that the proposed algorithm can obtain the best performance in terms of network lifetime, the energy consumption of $\mathrm{CHs}$, and network load balance.

\section{Competing interests}

The authors declare that they have no competing interests.

\section{Acknowledgements}

This work is partially supported by NSFC $(61379159,61201205,61371164)$, Chongqing Education Commission Science and Technology Research Project (KJ130513), Chongqing Basic and Cutting-edge Project (CSTC2013jcyjA40020, CSTC2011jjjq40002), Chongqing Graduate Research and Innovation Project (CYS14149).

\section{Author details \\ ${ }^{1}$ Engineering Research Center of Communication Network Testing Technologies, Chongqing University of Posts and Telecommunications, No. 2 Chongwen Rd, Nan'an Dist, Chongqing 400065, China. ${ }^{2}$ Chongqing Key Laboratory of Signal and Information Processing, Chongqing University of Posts and Telecommunications (CQUPT), No. 2, Chongwen Rd, Nan'an Dist, Chongqing 400065, China.}

Received: 14 January 2015 Accepted: 8 April 2015

Published online: 30 April 2015

\section{References}

1. D Cavalcanti, S Das, JF Wang, K Challapali, in Proceedings of 17th International Conference on Computer Communications and Networks. Cognitive radio based wireless sensor networks, (2008), pp. 1-6

2. B Akan, OB Karli, O Ergul, Cognitive radio sensor networks. IEEE Netw. 23(4), 34-40 (2009)

3. KLA Yau, P Komisarczuk, PD Teal, in IEEE 34th Conference on Local Computer Networks. Cognitive radio-based wireless sensor networks: conceptual design and open issues, (2009), pp. 955-962

4. GP Joshi, SY Nam, SW Kim. Cognitive radio wireless sensor networks: applications, challenges and research trends.SENSORS. 13(9), 11196-11228 (2013)

5. B Mamalis, D Gavalas, C Konstantopoulos, G Pantziou, Clustering in wireless sensor networks.RFID and Sensor Networks: Architectures, Protocols. Security and Integrations, 324-353 (2009)
6. ZY H Z Zhang, Zhang, Yuen Chau, Energy-efficient spectrum-aware clustering for cognitive radio sensor networks. Chinese Sci. Bull. 57(28-29), 3731-3739 (2012)

7. M Youssef, M Ibrahim, M Abdelatif, L Chen, AV Vasilakos.Routing metrics of cognitive radio networks: a survey. IEEE Commun. Surv. Tutorials. 16(1), 92-109 (2014)

8. HG T G Jiang, AV Wang, Vasilakos, QoE-driven channel allocation schemes for multimedia transmission of priority-based secondary users over cognitive radio networks. IEEE J. Selected Areas Commun. 30(7), 1215-1224 (2012)

9. SS Byun, I Balashingham, AV Vasilakos, HN Lee, Computation of an equilibrium in spectrum markets for cognitive radio networks. IEEE Trans. Comput. 63(2), 304-316 (2014)

10. F Akyildiz, WY Lee, MC Vuran, S Mohanty. Next generation dynamic spectrum access/cognitive radio wireless networks: a survey.Comput Netw. 13(50), 2127-2159 (2006)

11. N Chilamkurti, S Zeadally, A Vasilakos, V Sharma, Cross-layer support for energy efficient routing in wireless sensor networks. J. Sensors. 2009 (2009)

12. K Lin, JJPC Rodrigues, HW Ge, NX Xiong, XD Liang, Energy efficiency QoS assurance routing in wireless multimedia sensor networks. IEEE Syst. J. 5(4), 495-505 (2011)

13. HJ Cheng, ZHSu R Guo, NX Xiong, WZ Guo, Service-oriented node scheduling schemes with energy efficiency in wireless sensor networks. Int. J. Distributed Sensor Netw. 2014 (2014)

14. H Zhang, Z Zhang, H Dai, R Yin, XM Chen, in IEEE Global Telecommunications Conference. Distributed spectrum-aware clustering in cognitive radio sensor networks, (2011), pp. 1-6

15. RM Eletreby, HM Elsayed, MM Khairy, in International Conference on Cognitive Radio Oriented Wireless Networks and Communications. CogLEACH: a spectrum aware clustering protocol for cognitive radio sensor networks, (2014), pp. 179-184

16. M Ozger, OB Akan, in Proceedings IEEE INFOCOM. Event-driven spectrum-aware clustering in cognitive radio sensor networks, (2013), pp. 1483-1491

17. CF Li, M Ye, GH Chen, J Wu, in IEEE International Conference on Mobile Ad hoc and Sensor Systems. An energy-efficient unequal clustering mechanism for wireless sensor networks, (2005), pp. 604-612

18. AO Bicen, $O B$ Akan, Reliability and congestion control in cognitive radio sensor networks. Ad Hoc Netw. J. (Elsevier). 9(7), 1154-1164 (2011)

19. B Gulbahar, OB Akan.Information theoretical optimization gains in energy adaptive data gathering and relaying in cognitive radio sensor networks. IEEE Trans. Wireless Commun. 11(5), 1788-1796 (2012)

20. GA Shah, VC Gungor, OB Akan, A cross-layer QoS-aware communication framework in cognitive radio sensor networks for smart grid applications. IEEE Trans. Ind. Inf. 9(3), 1477-1485 (2013)

21. GA Shah, OB Akan, Performance analysis of CSMA-based opportunistic medium access protocol in cognitive radio sensor networks. Ad Hoc Netw. J. (Elsevier). 15, 4-13 (2014)

22. GA Shah, F Alagoz, E Fadel, OB Akan, A spectrum-aware clustering for efficient multimedia routing in cognitive radio sensor networks. IEEE Trans. Vehicular Technol. 63(7), 3369-3380 (2014)

23. GA Shah, OB Akan, Cognitive adaptive medium access control in cognitive radio sensor networks. IEEE Trans. vehicular Technol. 64(2), 757-767 (2015)

24. V Esmaeelzadeha, R Berangia, ES Hosseinia, OB Akanb, Stochastic backlog and delay bounds of generic rate-based AIMD congestion control scheme in cognitive radio sensor networks, (2015). Pervasive and Mobile Computing

25. W Heinzelman, A Chandrakasan, H Balakrishnan, An applicationspecific protocol architecture for wireless microsensor networks. IEEE Trans. Wireless Commun. 1(4), 660-670 (2002)

26. GA Shah, OB Akan, in IEEE International Conference on Communications. Spectrum-aware cluster-based routing for cognitive radio sensor networks, (2013), pp. 2885-2889 